\title{
Determinants of COVID-19 Vaccination Willingness among Health Care Workers: A Quick Online Survey in India
}

\author{
Rajesh Kumar ${ }^{1 *}$, Kalpana Beniwal ${ }^{2}$, Yogesh Bahurupi ${ }^{3}$, Ravi Kant ${ }^{4}$, Mukesh Bairwa ${ }^{5}$ \\ 'Department of Nursing, All India Institute of Medical Sciences (AlIMS), Rishikesh, India \\ ${ }^{2}$ Department of Nursing, Assistant Nursing Superintendent, All India Institute of Medical Sciences (AlIMS), Rishikesh, India \\ ${ }^{3}$ Department of Community \& Family Medicine, All India Institute of Medical Sciences (AIIMS), Rishikesh, India \\ ${ }^{4}$ Division of Diabetic and Metabolism, Department of Internal Medicine, All India Institute of Medical Sciences (AlIMS), Rishikesh, India \\ ${ }^{5}$ Department of Internal Medicine, All India Institute of Medical Sciences (AlIMS), Rishikesh, India
}

\section{See editorial commentary page on 411}

Background: The coronavirus disease 2019 (COVID-19) pandemic has caused a large number of deaths along with severe socio-economic effects. The vaccine is considered to be the last hope to control viral transmission. This study aimed to explore the determinants of health care workers' (HCWs) willingness to take the COVID-19 vaccination.

Methods: A structured, pre-validated, and pre-tested questionnaire was administered online to 599 HCWs including physicians, residents, and nurses from different types of healthcare set-ups across India. Information was collected regarding vaccine acceptability, attitude toward vaccination, and reasons for hesitancy. The chi-square test, followed by multinomial regression analysis, was applied to determine the factors associated with HCWs' vaccination willingness.

Results: It was found that $73 \%(\mathrm{n}=437)$ of HCWs were willing to accept the vaccines, while $10.85 \%(\mathrm{n}=65)$ refused and $16.2 \%(\mathrm{n}=96)$ needed more time to decide. Gender $(\mathrm{P}<0.001)$, occupation $(\mathrm{P}=0.040)$, working as front-line workers $(\mathrm{P}=0.008)$, vaccine manufacturing country preferences $(\mathrm{P}<0.001)$, and perceived risk of catching COVID-19 in the next 6 months $(\mathrm{P}=0.005)$ had a significant association with intent to receive vaccination (the response were "yes" vs. "no" and "not sure"). The reasons for vaccine hesitancy were vaccine safety and efficacy concerns, antivaccine attitude and beliefs, personal choice, and not wanting to take a vaccine before others.

Conclusion: The majority of HCWs agreed to take COVID-19 vaccines once available. Nevertheless, providing support to manage evolving vaccine environments will help change the perception of HCWs who refuse or are reluctant to take the vaccines.

Keywords: Vaccines; COVID-19; Health Personnel; Coronavirus; Vaccination Willingness

Received: March 31, 2021, Revised: May 14, 2021, Accepted: May 20, 2021

*Corresponding Author: Rajesh Kumar https://orcid.org/0000-0002-7504-5620

Tel: +91-7055911523, Fax: +91-135-2462983, E-mail: rajesh.nur@aiimsrishikesh.edu.in 


\section{INTRODUCTION}

The first case of severe acute respiratory syndrome coronavirus-2 was reported in Wuhan city, Hubei province, China, in the last week of December 2019. ${ }^{1)}$ Due to its rapid spread worldwide, the World Health Organization declared COVID-19 a Public Health Emergency of International Concern on January 30, 2020, and a pandemic on March 11, $2020{ }^{2)}$ Rapid transmission of the virus forced nations to take unprecedented measures to reduce the number of cases and slow down transmission. ${ }^{3)}$ As of March 29, 2021, the virus had reached 219 countries worldwide, infected 127 million people, and killed 2,797,695 people (https://www.worldometers.info/coronavirus/). Social distancing and quarantine measures helped reduce the transmission to an extent and flatten the epidemic curve. ${ }^{4)}$ However, these measures are neither practical nor sufficient to bring the pandemic to an end. After exposure to a large subset of the population or vaccination, developing herd immunity is the only effective measure to curb the infection. ${ }^{5)}$

The critical need for a vaccine to save humanity from the deadly virus cannot be overstated. Vaccination is the only way to control the epidemic. There are as many as 64 ongoing clinical trials, 20 of which are in the final stage of approval for use in humans. Likewise, 85 vaccines are under investigation in animals. ${ }^{6}$ Immunization has been found to be a successful way to decrease the global burden of disease and mortality. However, it would not be an exaggeration to say that vaccine hesitancy and refusal has contributed to vaccine delay and sometimes disease outbreaks. ${ }^{7)}$ Addressing the attitudes of understanding, acceptance (passive acceptance to active demand), ${ }^{8}$ resistance (total refusal to take a vaccine), and hesitancy (delay or uncertainty about taking a vaccine) toward COVID-19 vaccines is, therefore, a potentially crucial step to make the vaccination drive a big success. ${ }^{9,10)}$

Earlier research on vaccine resistance and hesitancy reported the explicit reason for refusal to accept an individual vaccine or program. ${ }^{5,6,9)}$ The role of health care workers (HCWs) is considered pivotal in educating their patients and other populations regarding vaccines. Healthcare professionals are seen as a reliable source of medical information and vaccine-related information as well. Health professionals who are resistant and show hesitancy could weaken public trust in the safety and efficacy of the vaccine and potentially reduce vaccine acceptability among the general population. ${ }^{10,11)}$ Previous research on public acceptance of COVID-19 vaccination revealed a mix of responses, mostly leaning toward hesitancy/resistance. ${ }^{6,10-13)}$ A report from the United Kingdom and Ireland reported 31\% and 35\% resistance and hesitancy among health professionals, respectively. ${ }^{6)}$ Similarly, an Israeli survey reported that health workers were highly skeptical about the safety of a vaccine developed in a very short period of time.

\section{METHODS}

\section{Study Design and Participants}

A rapid online survey was conducted from February 17, 2021, to March 1, 2021, using Google Forms through WhatsApp, Google plat- form (Gmail), Research Gate, and Facebook. The authors distributed the survey forms to $802 \mathrm{HCWs}$ including physicians, residents, nurses, and trainee nursing and medical students, who were given a period of 2 weeks to fill the survey form, with frequent reminders on the respective online platforms. Finally, 610 HCWs (response rate 76.06\%) working and training in different types of health care facilities responded to the survey within the time limit. Of these, 599 survey forms were used in the final analysis after discarding the forms with irrelevant or incomplete responses to questions related to sociodemographic variables.

\section{Measures and Instruments}

\section{1) Socio-demographic sheet}

A self-developed structured sheet was used to collect information on personal and professional characteristics such as age, gender, marital status, education, occupation, religion, family monthly income (Indian rupee), source of information on the vaccine, the experience of caring for a COVID-19 patient, type of healthcare facility employed at, professional expertise, and experience of treating or caring for patients with severe acute respiratory syndrome, Middle East respiratory syndrome coronavirus, or swine flu or Nipah virus. Experts in nursing, medicine, and infectious diseases validated the profile. The profile was pretested among eligible populations before its final use.

\section{2) Vaccination willingness and hesitancy measurement}

Participants were asked about their intention to receive vaccination. Vaccination willingness was queried by using a single question, "If a COVID-19 vaccine is available, I would take it," followed by the response options: "yes," "no," and "not sure." HCWs hesitating to take the COVID-19 vaccine (i.e., those who responded "no" or "not sure") were asked to select from a list of reasons for vaccine hesitancy. The options included novelty and rushed development of the vaccine, religious beliefs, personal choice (it was out of reason for vaccine hesitancy and considered as personal choice for no reason), fear of vaccines, safety concerns, lack of information about vaccine ingredients, a negative past experience with a vaccine, rumors of side effects, allergy to the vaccine, lack of trust in the government regarding the severity of COVID-19, skepticism regarding the vaccine's efficacy against a mutating virus, and prior exposure to COVID-19 infection..$^{14,15)}$ Additionally, respondents were asked about their perceived risk of catching the coronavirus infection in the next 6 months considering exposure to the virus and development of herd immunity in subsequent months. The participants' general awareness of facts related to the vaccine was also evaluated. They were asked about the availability of vaccines in India, whether and which Indian manufacturing pharmaceutical companies were working on vaccines, whether any other existing vaccines were being used as replacements for the COVID-19 vaccines, and the role of herd immunity in controlling the pandemic. 


\section{3) Attitude measurement}

The questionnaire consists of 12 items concerning different aspects of the COVID-19 vaccine and other related aspects of vaccination. A 5-point Likert scale ranging from strongly agree (5) to strongly disagree (1) was used to measure attitudes toward COVID-19 vaccination. The attitude towards a vaccine and potential vaccination was assessed using the statements-(1) COVID-19 is a self-limiting disease and so vaccination is unnecessary. (2) Vaccines should be available free of cost. (3) Approved vaccines will be safe to use. (4) Vaccines like bacille Calmette-Guérin (BCG) will be helpful to prevent COVID-19. (5) Vaccines enhance immunity and prevent infection. (6) Vaccination of a large population will control the disease by developing herd immunity. (7) Side effects of the vaccines are likely to be worse than the disease itself. (8) Vaccination is not required for every person. (9) I could get COVID-19 infection despite taking a vaccine. (10) Vaccination is important to end the pandemic. (11) Children and older people are more in need of vaccines. (12) I would rather build my immunity to prevent COVID-19 than take a vaccine. The questionnaire was validated with the help of experts in microbiology, nursing, and infectious diseases. The scale's reliability was computed using Cronbach's $\alpha$ and was found to be 0.87 for this study. ${ }^{16}$ )

\section{Sample Size}

The sample size was calculated considering the total population of HCWs in India (approximately 22,000,000) using the following formula: $[\operatorname{DEFF} \times N p(1-p)] /[(d 2 / Z 21-\alpha / 2) \times(N-1)+p \times(1-p)]=385$ for the final study; however, the authors decided to cover a large sample to extrapolate the findings throughout the country. The final sample size was 599 respondents.

\section{Data Analysis}

Data were transferred to a Microsoft Excel sheet (Microsoft Corp., Redmond, WA, USA) and analyzed using descriptive and inferential statistics, as appropriate. Frequencies and percentages were used to describe participant characteristics. The chi-square test was used to determine the association of participants' attributes with the potential intention to receive vaccination. Multivariable multinomial regression was applied to compute the unadjusted association of participant characteristics with intention to receive vaccination: yes, no, and not sure. The multinomial logistic regression results were presented as odds ratios (ORs) with a 95\% confidence interval (CI). Participants' characteristics showed a significant association with intention to receive vaccination and were included in multinomial modeling. The model performance was used by applying the Cox \& Snell and Nagelkerke statistics separately. The analysis was completed using IBM SPSS Statistics for Window ver. 23.0 (IBM Corp., Armonk, NY, USA). P-value $<0.05$ was considered significant for testing all statistical tests (two-sided).

\section{Ethical Consideration}

All India Institute of Medical Sciences (AIIMS) examined the detailed proposal and provided permission to conduct the survey (AIIMS/ IEC/21/66). In the online survey, respondents were asked to provide consent before they could participate. The participation was anonymous; no personal information was asked for, to protect the participants' privacy and confidentiality.

\section{RESULTS}

\section{Participants Characteristics}

Table 1 presents the participants' characteristics. Approximately twothirds $(72.95 \%)$ of HCWs were willing to accept the vaccines, while $10.85 \%$ refused and $16.2 \%$ were unsure and had not taken a decision yet. Participants over 30 years of age were more willing (74.95\%) to accept the vaccination than HCWs equal to or younger than 30 years (71.31\%); however, the association was not significant. Male HCWs $(79.52 \%)$ were more interested in taking vaccines than females (66.67\%). A substantial number of HCWs (51.08\%) had completed a bachelor's degree, and $41.74 \%$ had a master's degree. Interestingly, the specific occupations of HCWs showed significant associations with intention to vaccinate $(\mathrm{P}=0.040)$ - a substantial number of physicians were interested in getting vaccinated ( $81.33 \%)$, followed by nursemidwives $(74.81 \%)$, and trainee nursing and medical students (66.25\%). Surprisingly, residents were the least inclined (58.18\%) to accept the vaccine. Aversion to vaccination in residents might be due to increased anti-vaccination rhetoric with claims of vaccines being unsafe, the vaccine misinformation infodemic, and lack of trust in government policies. The proportion of HCWs with equal to or less than 6 years of professional experience was greater (56.59\%) among the survey participants. Front-line HCWs were found to be more willing (78.14\%) to take vaccines than others who were not directly involved in COVID-19 patient care $(\mathrm{P}=0.008)$. Notably, most of the respondents $(68.28 \%)$ had never dealt with a pandemic in their professional career before.

More than three-fourths (78.80\%) of the participants reported that their family members had not tested positive. Interestingly, intention to get vaccinated was significantly affected $(\mathrm{P}<0.001)$ by which country had manufactured the vaccine- $-79.74 \%$ of the respondents preferred vaccines made in India. Further, a higher perceived risk of COVID-19 infection in the coming 6 months had a significant association $(\mathrm{P}=0.005)$ with vaccination intention. Interestingly, HCWs who perceived light risk of infection in the coming 6 months showed more willingness (80.35\%) to receive the vaccine than the other groups who thought they may get a deadly attack of the virus (76.47\%) or had no chance of catching the virus $(72.43 \%)$.

\section{Acceptance and Vaccine Hesitancy}

Regarding vaccine hesitancy, HCWs were worried about the safety and efficacy of vaccines $(62.17 \%)$ and distrusted the government and pharmaceutical companies (19.38\%). Some said it was a matter of personal choice (15.63\%) or they did not want to take the vaccine before others (16.25\%). Surprisingly, it was seen that despite being healthcare pro- 
Table 1. Intention to potential vaccination and socio-demographic characteristics of health care workers $(\mathrm{N}=599)$

\begin{tabular}{|c|c|c|c|c|c|}
\hline \multirow{2}{*}{ Characteristic } & \multirow{2}{*}{ Total } & \multicolumn{3}{|c|}{ Intention to potential vaccination } & \multirow{2}{*}{ P-value } \\
\hline & & Yes & No & Not sure & \\
\hline Age (y) & & & & & 0.559 \\
\hline$\leq 30$ & $324(54.09)$ & 231 (71.31) & $36(11.11)$ & $57(17.60)$ & \\
\hline$>30$ & $275(45.90)$ & 206 (74.95) & $29(10.54)$ & $40(14.51)$ & \\
\hline Mean $\pm S D$ & $31.50 \pm 7.74$ & & & & \\
\hline Gender & & & & & $<0.001$ \\
\hline Male & $293(48.91)$ & 233 (79.52) & $30(10.24)$ & $30(10.24)$ & \\
\hline Female & 306 (51.09) & $204(66.67)$ & $35(11.44)$ & $67(21.90)$ & \\
\hline Qualification & & & & & 0.348 \\
\hline Diploma in nursing & $43(7.18)$ & 36 (83.72) & $3(6.98)$ & $4(9.30)$ & \\
\hline Bachelor degree & $306(51.08)$ & $182(59.48)$ & $31(10.13)$ & $37(12.09)$ & \\
\hline Master degree & $250(41.74)$ & 219 (87.61) & $31(12.41)$ & $56(22.40)$ & \\
\hline Type of health care setting & & & & & 0.334 \\
\hline Government & $490(81.80)$ & $363(74.08)$ & $55(11.22)$ & $72(14.69)$ & \\
\hline Private & $52(8.68)$ & 50 (96.15) & $7(13.46)$ & $17(32.69)$ & \\
\hline Semi-government & 57 (9.52) & $24(42.11)$ & $3(5.77)$ & $8(14.04)$ & \\
\hline Occupation & & & & & $0.040^{*}$ \\
\hline Physician & 75 (12.52) & 61 (81.33) & $7(9.33)$ & 7 (9.33) & \\
\hline Resident & $55(9.18)$ & $32(58.18)$ & $8(14.55)$ & $15(27.27)$ & \\
\hline Nurse/midwife & 389 (64.94) & 291 (74.81) & $37(9.51)$ & $61(15.68)$ & \\
\hline Students ${ }^{\dagger}$ & 80 (13.36) & 53 (66.25) & $13(16.25)$ & $14(17.50)$ & \\
\hline Working experience (y) & & & & & 0.373 \\
\hline$\leq 6$ & 339 (56.59) & 244 (71.98) & $34(10.03)$ & $61(17.99)$ & \\
\hline$>6$ & 176 (29.38) & 128 (72.72) & 23 (13.09) & $25(14.20)$ & \\
\hline Mean \pm SD & $7.34 \pm 6.76$ & & & & \\
\hline Median (IQR) & $6(2-10)$ & & & & \\
\hline Living place & & & & & 0.265 \\
\hline City & $402(67.11)$ & 301 (74.88) & $43(10.70)$ & $58(14.43)$ & \\
\hline Town & $134(22.37)$ & $90(67.16)$ & $18(13.43)$ & $26(19.40)$ & \\
\hline Village & 63 (10.52) & 46 (73.02) & $4(6.35)$ & $13(20.63)$ & \\
\hline Frontline workers & & & & & $0.008^{*}$ \\
\hline Yes & 311 (51.92) & $243(78.14)$ & 35 (11.25) & 59 (18.97) & \\
\hline No & $288(48.08)$ & $194(67.36)$ & $30(10.42)$ & $38(13.19)$ & \\
\hline Experience of caring/treating similar pandemic ${ }^{\ddagger}$ & & & & & 0.428 \\
\hline Yes & 190 (31.72) & 145 (76.32) & $19(10.00)$ & $26(15.26)$ & \\
\hline No & 409 (68.28) & $292(71.39)$ & $46(11.25)$ & $71(17.36)$ & \\
\hline Family members testes COVID-19 & & & & & 0.573 \\
\hline Yes & $127(21.20)$ & 88 (66.29) & $16(12.60)$ & $23(18.11)$ & \\
\hline No & $472(78.80)$ & $349(73.94)$ & $49(10.38)$ & $74(15.68)$ & \\
\hline Vaccine producing country preferences & & & & & $<0.001$ \\
\hline India made & 469 (78.30) & $374(79.74)$ & $39(8.31)$ & $56(11.94)$ & \\
\hline Outside India & $130(21.70)$ & $63(48.61)$ & $26(20.00)$ & $41(31.54)$ & \\
\hline Hospital working area & & & & & 0.235 \\
\hline COVID-19 area & $167(27.88)$ & $130(77.84)$ & $14(8.38)$ & $23(13.77)$ & \\
\hline Non-COVID area & $432(72.12)$ & $307(71.06)$ & $51(11.81)$ & $74(17.13)$ & \\
\hline Perceived risk of corona attack in next 6 months & & & & & $0.005^{\star}$ \\
\hline I think I had COVID-19 & $166(27.71)$ & $109(65.66)$ & $26(15.66)$ & $31(18.67)$ & \\
\hline Will not get the infection & $243(40.57)$ & $176(72.43)$ & $20(8.23)$ & $47(19.34)$ & \\
\hline Only light infection & $173(28.89)$ & 139 (80.35) & $15(6.17)$ & 19 (10.98) & \\
\hline Deadly infection attack & $17(2.84)$ & $13(76.47)$ & $4(23.53)$ & 0 & \\
\hline I am willing to accept the COVID-19 vaccine & $599(100.00)$ & 437 (72.95) & $65(10.85)$ & $97(16.2)$ & \\
\hline
\end{tabular}

Values are presented as number (\%), mean $\pm \mathrm{SD}$, or median (IQR), unless otherwise stated.

SD, standard deviation; IQR, interquartile range; COVID-19, coronavirus disease 2019.

${ }^{*} \mathrm{P}<0.05$. ${ }^{\dagger}$ Nursing \& medical students. ${ }^{\ddagger}$ Severe acute respiratory syndrome, Middle East respiratory syndrome, or swine-flu or Nipah virus. 


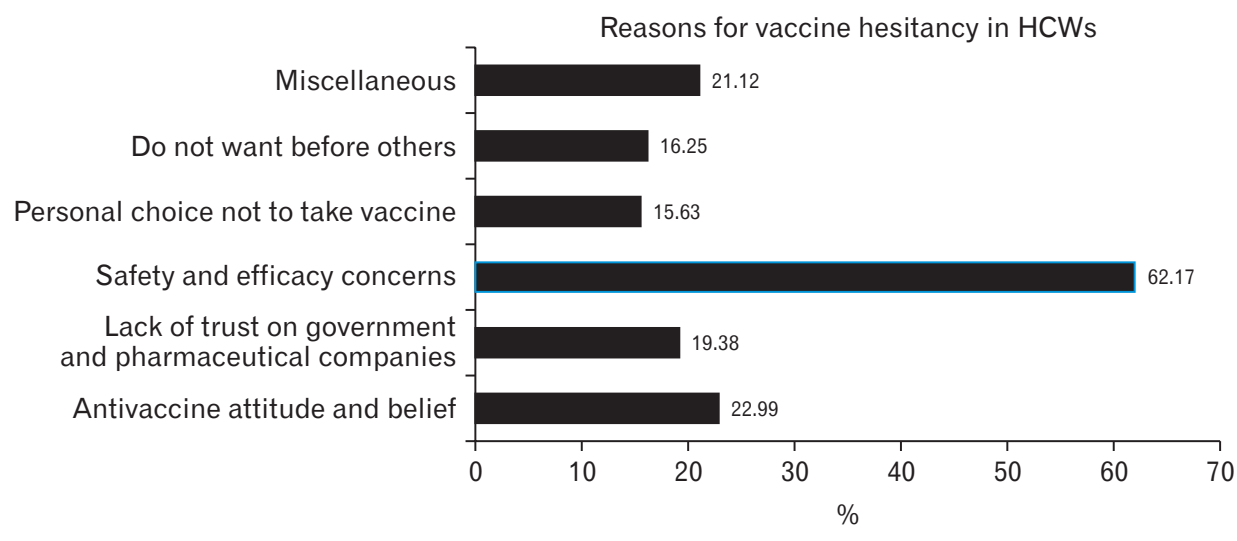

Figure 1. Reasons for vaccine hesitancy among health care workers (HCWs).

Self-limiting disease: no need for a vaccine. Vaccine should also made free of cost. Approved vaccines will be safe.

Existing vaccines (BCG, etc.) will help prevent coronavirus.

Vaccine will enhance immunity and prevent infection. Vaccinating large population will help to control the pandemic. Side-effects of the vaccine are likely to be worse than COVID-19.

Corona vaccine does not require for everyone. I will get COVID infection after taking the vaccine. Vaccine is important to end pandemic. Children and older people are more in need of vaccines. I would rather build immunity by exposure to an infection.

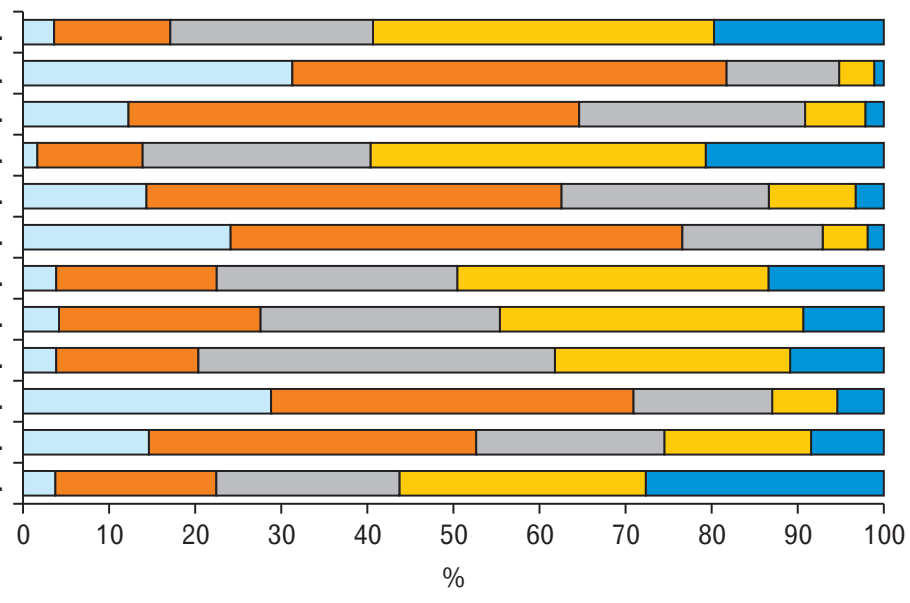
Strongly agree
Agree
Neutral
Disagree
Strongly disagree

Figure 2. The attitude of health care workers towards coronavirus disease 2019 (COVID-19) vaccines. BCG, bacille Calmette-Guérin.

fessionals, a considerable number of respondents (22.99\%) had antivaccine attitudes and beliefs (Figure 1).

The attitude of HCWs toward vaccines was measured using a 5-point Likert scale. For better presentation, the findings of agreement (strongly agree+agree) and disagreement (strongly disagree+disagree) are presented here.

More than half of the participants agreed that approved vaccines would be safe to use $(64.81 \%)$ and help to control the pandemic (90.02\%); however, $12.82 \%$ and $9 \%$ disagreed with the statements that vaccination is important to control pandemics and vaccines are safe to use, respectively. Further, $62.72 \%$ of the respondents believed that vaccines provide immunity and are essential to end this pandemic (71.01\%). Most respondents (82.0\%) felt that older adults and children need vaccination and that they should receive vaccines free of cost (50.63\%). Meanwhile, $59.24 \%$ disagreed with the statement that COVID-19 is a self-limiting disease and everyone does not need to be vaccinated (44.54\%). Over half of them (59.44\%) were not in favor of using the BCG vaccine as a substitute for a COVID-19 vaccine to help prevent the coronavirus pandemic. Further, $56.01 \%$ were not in favor of exposing themselves to an infection to develop immunity against the virus. Likewise, $49.42 \%$ of participants disagreed that vaccines' side effects were likely to be worse than COVID-19 itself (Figure 2).

The multinomial regression model helped determine the strength of association between participant characteristics and vaccination intention. Findings indicated that male HCWs were significantly more inclined (OR, 0.388; 95\% CI, 0.226-0.666; $\mathrm{P}<0.05$ ) to accept the vaccine than females. Furthermore, residents were three-fold more unwilling or in a dilemma (OR, 3.479; 95\% CI, 1.287-9.408; $\mathrm{P}<0.05)$ to accept the vaccine than trainee nursing and medical students. An obvious cause of this hesitancy is falsehoods about vaccines spread by antivaccine campaigners which were amplified by the social media. Similarly, HCWs who believed that they had already been exposed to the coronavirus without falling sick (due to a strong immune system) had a two-fold higher risk of not accepting (OR, 2.192; 95\% CI, 1.117-4.300; $\mathrm{P}<0.05)$ or being on the fence (OR, 2.974; 95\% CI, 1.517-5.829; $\mathrm{P}<0.05$ ) regarding whether to accept the vaccine or not once it was available. Likewise, HCWs who predicted no infection risk exhibited a 2-time higher (OR, 2.070; 95\% CI, 1.454-5.014; $\mathrm{P}<0.05)$ dilemma regarding vaccination than the group that expected to get infected in the next 6 months. Participants involved in the direct care of COVID-19 patients 
were less uncertain (OR, 0.501; 95\% CI, 0.293-0.877; $\mathrm{P}<0.05)$ than another group of HCWs not involved in direct COVID-19 patient care. Interestingly, HCWs showed significantly lower interest in receiving the vaccine produced in India ( $\mathrm{OR}, 0.268 ; 95 \% \mathrm{CI}, 0.149-0.482 ; \mathrm{P}<0.001$ ) and willingness to a lesser extent (OR, 0.215; 95\% CI, 0.127-0.363; $\mathrm{P}<0.001)$ to accept the vaccines than vaccines made outside the country (Table 2).

\section{DISCUSSION}

The government has taken unprecedented measures to control the spread of COVID-19. However, vaccination is considered to be a ray of hope to control the pandemic. In this survey, HCWs' opinions were evaluated for vaccination willingness and reasons for vaccine hesitancy. This nationwide quick survey reported that $73 \%$ of the HCWs are willing to accept the vaccine, and about $27 \%$ preferred to defer their vaccination and wait to review more safety data. These results are in agreement with earlier similar research conducted in Hong Kong and Malta. Vaccination willingness among health professionals in Hong Kong $^{17)}$ and Malta ${ }^{18)}$ was slightly lower (63\% and 50\%, respectively) compared to that in the present study. Another study conducted in Turkey reported that $68.8 \%$ of health professionals were willing to get vaccinated. ${ }^{11)}$

In the present study, participants declared common reasons for vaccine hesitancy, including safety and efficacy concerns, possibility of side effects, antivaccine attitude, fear and phobia, and not wanting to take the vaccine before others. These reasons agree with previous studies that show health professionals' concerns regarding newly developed vaccines. A qualitative study with HCWs in Greece, Romania,
France, and Croatia suggested hesitancy among the study participants. Reasons included lack of trust in government and pharmaceutical companies manufacturing vaccines, safety and efficacy issues, the rushed development of vaccines, and possible side effects. ${ }^{10,11)}$ Likewise, homeopathy practitioners in Europe also deferred their vaccination. ${ }^{10)}$ Vaccine hesitancy among health workers is problematic because the general public views them as a reliable source of information regarding vaccine safety and efficacy, and their hesitancy may lead to strengthening of anti-vaccine sentiments among people.

The findings revealed that a greater number of male HCWs aged above 30 years are more interested in taking a vaccine. In a study conducted in Canada, men aged above 60 years working in the tertiary care unit were more willing to take a vaccine. ${ }^{19)}$ Similarly, physicians in our study showed a higher willingness to accept vaccines, which is similar to the findings of a Hong Kong study. ${ }^{19)}$ In the present study, participants with a perceived higher risk of coronavirus infection in the near future showed greater vaccination willingness. Similarly, a Saudi Arabian study showed that participants working in intensive care units with apprehensions about infecting critical patients (who were being treated for other diseases) were more willing to take vaccines. ${ }^{19)}$ However, the fear of catching the COVID-19 infection and infecting family members did not affect their vaccination willingness. ${ }^{20)}$ In contrast, studies conducted in the United States reported a higher vaccination willingness among participants who thought they had had coronavirus disease and had developed immunity against it. ${ }^{21)}$ However, it is difficult to conclusively identify such differences in the findings. We can only speculate that studies done at different periods show a change in interest and willingness to accept vaccines. ${ }^{14,15)}$ Increasing vaccination willingness has significant benefits in controlling the pan-

Table 2. Characteristics of health care workers associated with the willingness to accept the COVID-19 vaccine: multivariable multinomial regression model (N=599)

\begin{tabular}{|c|c|c|c|c|}
\hline \multirow{2}{*}{ Variable } & \multicolumn{2}{|c|}{ Willingness to vaccine: no vs. yes } & \multicolumn{2}{|c|}{ Willingness to vaccine: not sure vs. yes } \\
\hline & $\mathrm{OR}(95 \% \mathrm{Cl})$ & SE & $\mathrm{OR}(95 \% \mathrm{Cl})$ & SE \\
\hline \multicolumn{5}{|l|}{ Gender } \\
\hline Male & $0.773(0.432-1.386)$ & 0.298 & $0.388(0.226-0.666)$ & $0.275^{\star}$ \\
\hline Female & Ref. & & Ref. & \\
\hline \multicolumn{5}{|l|}{ Occupation } \\
\hline Physician & $0.554(0.190-1.615)$ & 0.546 & $0.784(0.269-2.287)$ & 0.546 \\
\hline Resident & $1.193(0.392-3.630)$ & 0.568 & $3.479(1.287-9.408)$ & $0.508^{*}$ \\
\hline Nurses & $0.708(0.334-1.503)$ & 0.384 & $1.238(0.609-2.519)$ & 0.347 \\
\hline Students $^{\dagger}$ & Ref. & & Ref. & \\
\hline \multicolumn{5}{|c|}{ Risk of getting COVID-19 infection in the next 6 months } \\
\hline I think I had COVID-19 & $2.192(1.117-4.300)$ & $0.344^{*}$ & $2.974(1.517-5.829)$ & $0.343^{*}$ \\
\hline I think I will not get & $1.054(0.530-2.098)$ & 0.351 & $2.070(1.454-5.014)$ & $0.316^{*}$ \\
\hline I think I will get infection & Ref. & & Ref. & \\
\hline \multicolumn{5}{|l|}{ Direct involvement in patient care } \\
\hline Yes & $0.647(0.348-1.201)$ & 0.316 & $0.501(0.293-0.877)$ & $0.279^{*}$ \\
\hline No & Ref. & & Ref. & \\
\hline \multicolumn{5}{|c|}{ Vaccine producing country preferences } \\
\hline Made in India & $0.268(0.149-0.482)$ & $0.300^{\star \star *}$ & $0.215(0.127-0.363)$ & $0.0267^{\star \star \star}$ \\
\hline Outside India & Ref. & & Ref. & \\
\hline
\end{tabular}

COVID-19, coronavirus disease 2019; OR, odds ratio; Cl, confidence interval; SE, standard error; Ref., reference.

${ }^{\star} \mathrm{P}<0.05$. ${ }^{\star * \star} \mathrm{P}<0.001$. ${ }^{\dagger}$ Nursing \& medical. 
demic.

In our study, $10.85 \%$ of HCWs refused, and $16.2 \%$ were waiting to obtain more scientific information on safety and efficacy, which is available mainly in many scientific publications and could be considered a reason for the gradual increase in the acceptance of vaccines. ${ }^{22,23)}$ This also highlights the implications of the circulation of scientific information regarding the vaccines, which can play a crucial role in determining the vaccination willingness of participants. It is imperative to vaccinate a significant subset of populations to achieve herd immunity before natural immunity after infection. Targeting $70 \%$ of the population considering $100 \%$ efficacy will ensure substantial herd immunity to fight the battle against the coronavirus. ${ }^{24)}$

In this study, vaccination willingness was higher among frontline workers involved in direct patient care. This higher acceptance rate could be due to a higher perceived risk of contracting COVID-19. Likewise, this trend is also constant among HCWs and nurses having prolonged and more direct contact with COVID-19 patients and, therefore, at higher risk of getting infected. These findings mirror the trends observed in earlier work on HCWs involved in direct patient care, having higher odds of accepting the vaccine. ${ }^{19,21)}$

Recently, vaccines from India and Western countries claimed 70\%$95 \%$ efficacy against the coronavirus; this number could vary or higher for some vaccines. ${ }^{22,23)}$ Thus, it is of utmost importance for the government to vaccinate a large number of HCWs to prevent infection and protect the healthcare workforce. Previous studies have shown that vaccinating HCWs reduced absenteeism and mortality. ${ }^{21,25,26)}$

Coronavirus vaccination willingness was higher among advanced age male HCWs working in private health care institutions. This trend matches earlier Western studies that showed a higher vaccination willingness with increasing age. ${ }^{21)}$ However the association of age, clinical discipline, and profession with vaccination willingness has not been found to be significant. However, a study from China showed no difference in vaccination willingness between females and males. ${ }^{4}$ These findings are also similar to a study from London that indicated higher vaccination willingness among male HCWs. ${ }^{27)}$ The difference in the findings might be related to the heterogeneity of population among different studies and points to the need to pool data to reach a specific conclusion.

HCWs' attitudes toward vaccine safety and the importance of controlling the pandemic were favorable. However, HCWs were more concerned about providing vaccines to children and older populations considering that they are more vulnerable to infections. More than half of HCWs believe that vaccines should be made available free of cost to all, and not everyone needs to be vaccinated. The vaccine safety and efficacy trends uncovered in this study mirror earlier similar studies that reported high concern about the rapid and hurried development of vaccines and potential adverse effects. ${ }^{15,18,19,21)} \mathrm{A}$ lack of trust in government and pharmaceutical companies was found in this as well as earlier studies.

This study's significant strength is that it used a large representative sample including HCWs with diverse ages and educational back- grounds working in different healthcare setups across the country. However, an overwhelming response of HCWs in a short period enabled researchers to complete the survey quickly.

We acknowledge the limitations of this study. Considering the high transmission rate of COVID-19, an online survey was planned to avoid transmission and could be viewed as a limitation of this study. More HCWs working in the public health sector responded to the survey which could be considered as another potential source of bias in the study findings. The authors advise interpreting the findings considering social desirability bias, although the response was anonymized entirely to control for bias. The study findings on vaccination willingness and attitude may be influenced by media misinformation on the COVID-19 vaccines under development.

Most importantly, we completed the study at the time of the vaccination campaign launched solely for HCWs in India, and limited information was available on the safety and efficacy of the vaccines. Despite many limitations, the study findings provide new insights to address the concerns and issues raised by HCWs. Providing reliable information using trustable sources will improve vaccination willingness among HCWs.

Since the concerns of vaccine safety and efficacy among HCWs are important reasons for vaccine hesitancy, it is advisable to circulate information through regulatory bodies and medical or health care agencies to boost confidence among HCWs.

\section{CONFLICT OF INTEREST}

No potential conflict of interest relevant to this article was reported.

\section{ORCID}

Rajesh Kumar: https://orcid.org/0000-0002-7504-5620

Kalpana Beniwal: https://orcid.org/0000-0001-9161-2800

Yogesh Bahurupi: https://orcid.org/0000-0003-2433-1624

Ravi Kant: https://orcid.org/0000-0003-1144-4478

Mukesh Bairwa: https://orcid.org/0000-0002-8974-5775

\section{REFERENCES}

1. Zhu N, Zhang D, Wang W, Li X, Yang B, Song J, et al. A novel coronavirus from patients with pneumonia in China, 2019. N Engl J Med 2020; 382:727-33.

2. Lai J, Ma S, Wang Y, Cai Z, Hu J, Wei N, et al. Factors associated with mental health outcomes among health care workers exposed to coronavirus disease 2019. JAMA Netw Open 2020;3:e203976.

3. Kumar R, Singh V, Mohanty A, Bahurupi Y, Gupta PK. Corona healthcare warriors in India: knowledge, attitude, and practices during COVID-19 outbreak. J Educ Health Promot 2021;10:44.

4. Fu C, Wei Z, Pei S, Li S, Sun X, Liu P. Acceptance and preference for COVID-19 vaccination in health-care workers (HCWs). MedRxiv 2020 Jan 1 [Epub]. https://doi.org/10.1101/2020.04.09.20060103.

5. Paterson P, Meurice F, Stanberry LR, Glismann S, Rosenthal SL, Larson 
HJ. Vaccine hesitancy and healthcare providers. Vaccine 2016;34:67006.

6. Murphy J, Vallieres F, Bentall RP, Shevlin M, McBride O, Hartman TK, et al. Psychological characteristics associated with COVID-19 vaccine hesitancy and resistance in Ireland and the United Kingdom. Nat Commun 2021;12:29.

7. Larson HJ, Smith DM, Paterson P, Cumming M, Eckersberger E, Freifeld CC, et al. Measuring vaccine confidence: analysis of data obtained by a media surveillance system used to analyse public concerns about vaccines. Lancet Infect Dis 2013;13:606-13.

8. Habersaat KB, Jackson C. Understanding vaccine acceptance and demand-and ways to increase them. Bundesgesundheitsblatt Gesundheitsforschung Gesundheitsschutz 2020;63:32-9.

9. Larson HJ, Jarrett C, Eckersberger E, Smith DM, Paterson P. Understanding vaccine hesitancy around vaccines and vaccination from a global perspective: a systematic review of published literature, 20072012. Vaccine 2014;32:2150-9.

10. Kose S, Mandiracioglu A, Sahin S, Kaynar T, Karbus O, Ozbel Y. Vaccine hesitancy of the COVID-19 by health care personnel. Int J Clin Pract 2021;75:e13917.

11. Karafillakis E, Dinca I, Apfel F, Cecconi S, Wurz A, Takacs J, et al. Vaccine hesitancy among healthcare workers in Europe: a qualitative study. Vaccine 2016;34:5013-20.

12. Thoon KC, Chong CY. Survey of healthcare workers' attitudes, beliefs and willingness to receive the 2009 pandemic influenza A (H1N1) vaccine and the impact of educational campaigns. Ann Acad Med Singap 2010;39:307-6.

13. Dror AA, Eisenbach N, Taiber S, Morozov NG, Mizrachi M, Zigron A, et al. Vaccine hesitancy: the next challenge in the fight against COVID-19. Eur J Epidemiol 2020;35:775-9.

14. Fisher KA, Bloomstone SJ, Walder J, Crawford S, Fouayzi H, Mazor KM. Attitudes toward a potential SARS-CoV-2 vaccine: a survey of U.S. adults. Ann Intern Med 2020;173:964-73.

15. Malik AA, McFadden SM, Elharake J, Omer SB. Determinants of COVID-19 vaccine acceptance in the US. EClinicalMedicine 2020;26: 100495.

16. Taber KS. The use of Cronbach's alpha when developing and reporting research instruments in science education. Res Sci Educ 2018;48:1273-
96.

17. Kwok KO, Li KK, Wei WI, Tang A, Wong S, Lee SS. Editor's Choice: Influenza vaccine uptake, COVID-19 vaccination intention and vaccine hesitancy among nurses: a survey. Int J Nurs Stud 2021;114:103854.

18. Grech V, Gauci C, Agius S. Withdrawn: Vaccine hesitancy among Maltese healthcare workers toward influenza and novel COVID-19 vaccination. Early Hum Dev 2020 Oct 1 [Epub]. https://doi.org/10.1016/ j.earlhumdev.2020.105213.

19. Dzieciolowska S, Hamel D, Gadio S, Dionne M, Gagnon D, Robitaille L, et al. COVID-19 vaccine acceptance, hesitancy, and refusal among Canadian healthcare workers: a multicenter survey. Am J Infect Control 2021;49:1152-7.

20. Goodman JL, Grabenstein JD, Braun MM. Answering key questions about COVID-19 vaccines. JAMA 2020;324:2027-8.

21. Shekhar R, Sheikh AB, Upadhyay S, Singh M, Kottewar S, Mir H, et al. COVID-19 vaccine acceptance among health care workers in the United States. Vaccines (Basel) 2021;9:119.

22. Polack FP, Thomas SJ, Kitchin N, Absalon J, Gurtman A, Lockhart S, et al. Safety and efficacy of the BNT162b2 mRNA COVID-19 vaccine. N Engl J Med 2020;383:2603-15.

23. Voysey M, Clemens SA, Madhi SA, Weckx LY, Folegatti PM, Aley PK, et al. Safety and efficacy of the ChAdOx1 nCoV-19 vaccine (AZD1222) against SARS-CoV-2: an interim analysis of four randomised controlled trials in Brazil, South Africa, and the UK. Lancet 2021;397:99111.

24. Fontanet A, Cauchemez S. COVID-19 herd immunity: where are we? Nat Rev Immunol 2020;20:583-4.

25. Lemaitre M, Meret T, Rothan-Tondeur M, Belmin J, Lejonc JL, Luquel L, et al. Effect of influenza vaccination of nursing home staff on mortality of residents: a cluster-randomized trial. J Am Geriatr Soc 2009;57: $1580-6$.

26. Murti M, Otterstatter M, Orth A, Balshaw R, Halani K, Brown PD, et al. Measuring the impact of influenza vaccination on healthcare worker absenteeism in the context of a province-wide mandatory vaccinateor-mask policy. Vaccine 2019;37:4001-7.

27. Larson HJ, Bhutta ZA. Security, insecurity, and health workers: the case of polio. JAMA Intern Med 2013;173:1393-4. 